Published in final edited form as:

Science. 2014 January 31; 343(6170): 496-497. doi:10.1126/science.1250171.

\title{
Pathogen Specialization
}

\author{
Gitta Coaker \\ Department of Plant Pathology, University of California, Davis, Davis, CA 95616, USA \\ Gitta Coaker: glcoaker@ucdavis.edu
}

\section{Abstract}

The ability to infect new hosts can drive the evolution and specialization of secreted pathogen proteins.

Plants can be attacked by a vast range of pathogen classes, causing substantial agricultural losses. The Phytophthora (meaning "plant killer") genus is a particularly destructive pathogen that causes root and stem base decay in a wide range of plants. Phytophthora infestans, which precipitated the Irish potato famine, originated in Central Mexico and is closely related to other Phytophthora species with distinct host ranges $(1,2)$. Pathogen effectors that are secreted during infection play a key role in disease biology, but effectorinduced adaptation to new hosts is an understudied topic. On page 552 of this issue, Dong et al. investigate how Phytophthora effector proteins evolve the ability to specialize on new hosts (see the figure) (3).

Phytophthora is a genus of oomycetes that exhibit filamentous growth on plants. Oomycetes share some phenotypes with fungi, but are phylogenetically related to photosynthetic brown algae and are thought to have initially emerged from marine environments. The Phytophthora genus comprises 10 main lineages designated as clades 1 to 10 . Clade 1c, a subdivision of clade 1, includes $P$. infestans (infecting potato and tomato) and $P$. mirabilis (infecting 4 o'clock weeds), indicating that these species share a recent common ancestor (2). Genome comparisons between $P$. infestans and $P$. mirabilis highlight alterations and patterns of selection in repetitive DNA containing rapidly evolving families of virulence genes (such as effectors) (4).

The 82 effectors undergoing positive selection between $P$. infestans and $P$. mirabilis are promising candidates shaping host specialization (4). Dong et al. focused their efforts on the EPIC1 effector, which is abundantly secreted during infection of tomato and inhibits extracellular papain-like proteases (including RCR3) that are involved in plant immune perception (5).

Dong et al. now report that the P. mirabilis epiCl ortholog (PmepiCl) shows signatures of positive selection, suggesting that this effector has evolved to function in Mirabilis jalapa, the 4 o'clock plant, following the split between $P$. mirabilis and $P$. infestans. Using activitybased profiling with a probe that targets papain proteases, the authors demonstrate that recombinant EPIC1 from $P$. infestans effectively inhibits tomato and wild potato RCR3 proteases, whereas PmEPIC1 does not. The authors identify two PmEPIC1 M. jalapa targets with homology to RCR3 (MRP1 and MRP2). Subsequent experiments confirmed that MRP2 
is an active protease and can be effectively inhibited only by PmEPIC1. Thus, EPIC1 effectors function more effectively on their respective hosts, supporting the hypothesis of effector specialization after a host jump.

To define the biochemical basis of EPIC1 host specialization, Dong et al. inferred the ancestral allele of epiCl using maximum likelihood methods. Ancestral EPIC1 had similar specificity as $P$. infestans EPIC1 for RCR3. Thus, the ability of PmEPIC1 to function more effectively on MRP2 was likely acquired after host jumping.

Surprisingly, both EPIC1 and PmEPIC1 can bind MRP2 using co-immunoprecipitation. Competition experiments show that binding likely occurs at the active site of MRP2 for both EPIC1 and PmEPIC1. How can two similar EPIC1 effectors exhibit different target specificity while still binding to both effective and ineffective targets? Analyses of variant amino acid residues superimposed on an existing structure of tarocystatin in complex with papain protease (6) indicate that EPIC1 effectors have similar folds and likely bind proteases at three sites, two of which are polymorphic. A single amino acid polymorphism in PmEPIC1 and its corresponding protease, MRP2, determine specificity.

Future experiments are needed to determine whether PmEPIC1 provides a fitness advantage. If it does, then this would validate the importance of effector specialization for disease biology. These experiments are possible but likely to be difficult to interpret. Oomycete genomes contain large effector arsenals, with $\sim 563$ cell-entering effectors in P. infestans (7). Collectively, effectors are critical virulence factors, but each individual effector typically provides a minor fitness advantage for the pathogen (8). It is likely that numerous effectors together rather than individual effectors are crucial for fitness.

By exploring both evolutionary pressures and biochemical adaptation of EPIC1 and corresponding host proteases, Dong et al. reveal that selection for single amino acid polymorphisms can significantly affect effector specialization. These findings open an exciting new door to understanding the role effectors play in shaping pathogen adaptation. Conserved core effectors undergoing positive selection could be promising targets for disease control. Nature's ability to select for biochemical specialization has implications for engineering enzymes and their corresponding targets for enhanced specificity. Undoubtedly, many important secrets remain to be discovered.

\section{Acknowledgments}

G. C. is funded by the NIH and NSF. I regret not being able to cite some work due to space limitations.

\section{References and Notes}

1. Grünwald NJ, Flier WG. Annu Rev Phytopathol. 2005; 43:171. [PubMed: 16078881]

2. Blai JE, et al. PLOS ONE. 2012; 7:e37003. [PubMed: 22615869]

3. Dong S, et al. Science. 2014; 343:552. [PubMed: 24482481]

4. Raffaele S, Kamoun S. Nat Rev Microbiol. 2012; 10:417. [PubMed: 22565130]

5. Song J, et al. Proc Natl Acad Sci USA. 2009; 106:1654. [PubMed: 19171904]

6. Chu MH, et al. Planta. 2011; 234:243. [PubMed: 21416241]

7. Haas BJ, et al. Nature. 2009; 461:393. [PubMed: 19741609] 
8. Fabro G, et al. PLOS Pathog. 2011; 7:e1002348. [PubMed: 22072967] 


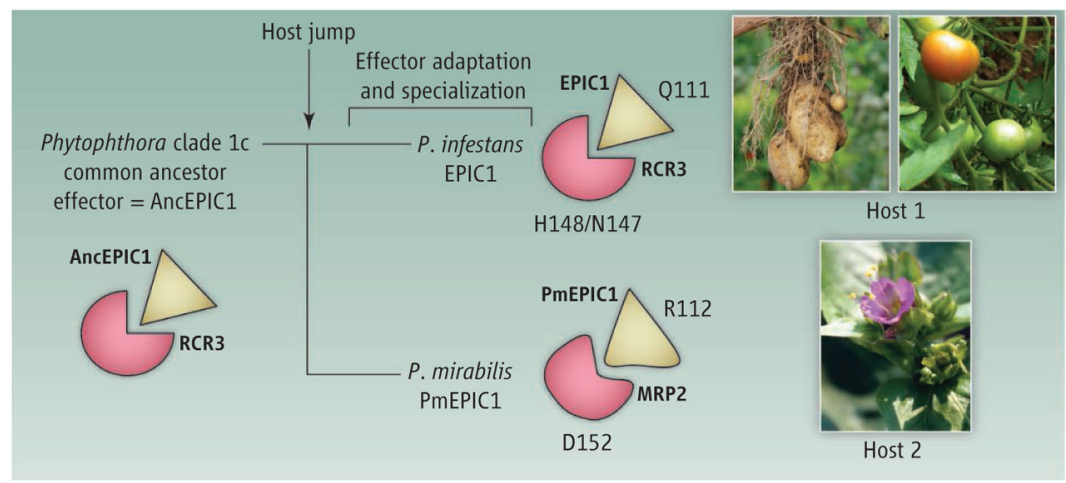

Figure. Route to specialization

A host jump within Phytophthora 1c led to the emergence of $P$. mirabilis, which can infect M. jalapa. Dong et al. show that effector specialization following the host jump is associated with the R112 mutation in PmEPIC1, enabling effective inhibition of the M. jalapa MRP2 protease. Polymorphic residues controlling specificity for the host and pathogen are highlighted. Adapted from fig. S18 in (3). 